\title{
A sufficient condition of regularity for axially symmetric solutions to the Navier-Stokes equations
}

\author{
G. Seregin and W. Zajaczkowski
}

\begin{abstract}
In the present paper, we prove a sufficient condition of local regularity for suitable weak solutions to the Navier-Stokes equations having axial symmetry. Our condition is an axially symmetric analog of the so-called $L_{3, \infty}$-case in the general local regularity theory.
\end{abstract}

1991 Mathematical subject classification (Amer. Math. Soc.): 35K, $76 \mathrm{D}$.

Key Words: the Navier-Stokes equations, axial symmetry, suitable weak solutions, backward uniqueness.

\section{Introduction}

In the present paper, we address the problem of regularity for axisymmetric solutions to the Navier-Stokes equations. In contrast to many others, see, for example, [7], [21], [9], [11],[12], [22], 23], and [24], we study this problem in local setting.

Our work is motivated by results of two different papers [2] and [4]. To explain that, we need the following simple notation. Let $e_{1}, e_{2}, e_{3}$ be an orthogonal basis of the Cartesian coordinates $x_{1}, x_{2}, x_{3}$ and $e_{\varrho}, e_{\varphi}, e_{3}$ be an orthogonal basis of the cylindrical coordinates $\varrho, \varphi, x_{3}$ chosen so that

$$
e_{\varrho}=\cos \varphi e_{1}+\sin \varphi e_{2}, \quad e_{\varphi}=-\sin \varphi e_{1}+\cos \varphi e_{2}, \quad e_{3}=e_{3} .
$$

Then, for any vector-valued field $v$, we have representations

$$
v=v_{i} e_{i}=v_{1} e_{1}+v_{2} e_{2}+v_{3} e_{3}=v_{\varrho} e_{\varrho}+v_{\varphi} e_{\varphi}+v_{3} e_{3} .
$$

The classical Navier-Stokes equations, which are invariant with respect to transformation of coordinates, have the form

$$
\partial_{t} v+v \cdot \nabla v-\Delta v+\nabla p=0, \quad \operatorname{div} v=0
$$


and are satisfied in some space-time domain. Here, as usual, $v$ and $p$ stand for the velocity field and the pressure field, respectively.

In our considerations, we always assume that $v_{\varrho}, v_{\varphi}, v_{3}$, and $p$ are independent of the polar angle $\varphi$. In [2], Chae and Lee consider the Cauchy problem for the Navier-Stokes equations under the above assumption on axial symmetry. In addition to usual conditions on the initial data, the authors of [2] assume that velocity field $v$ obeys

$$
\int_{0}^{T} d t\left(\int_{\mathbb{R}^{3}}|v|^{\gamma} d \varrho d x_{3}\right)^{\frac{\alpha}{\gamma}}<+\infty,
$$

with $1 / \alpha+1 / \gamma \leq 1 / 2,2<\gamma<+\infty, 2<\alpha \leq+\infty$, and prove the regularity of solutions to the Cauchy problem for (1.1) on time interval ]0,T[. In fact, they prove even more, their statement is still true if $|v|$ is replaced with $\sqrt{v_{\varrho}^{2}+v_{\varphi}^{2}}$. However, it remains unclear whether or not the regularity takes place in the marginal case $\gamma=2$ and $\alpha=+\infty$. To our opinion, the case cannot be treated by methods developed in paper [2] because, in a sense, it is an analog of the so-called $L_{3, \infty}$-case studied in [4]. In turn, $L_{3, \infty}$-case is marginal to the so-called Ladyzhenskaya-Prodi-Serrin condition, see [13], [19], 6], [20], [5], [16], and [17]. It seems quite reasonable to interpret the result of [2], see Theorem 3 there, as the Ladyzhenskaya-Prodi-Serrin condition for axially symmetric problems. To treat $L_{3, \infty}$-solutions in generic setting, one needs new technique based on backward uniqueness for the heat operator with variable lower order terms. In this, paper, we wish to extend this method to the axially symmetric case.

To formulate our main result, we introduce the additional notation:

$$
\begin{gathered}
\mathcal{C}\left(x_{0}, R\right)=\left\{x \in \mathbb{R}^{3} \| x=\left(x^{\prime}, x_{3}\right), x^{\prime}=\left(x_{1}, x_{2}\right),\right. \\
\left.\left|x^{\prime}-x_{0}^{\prime}\right|<R,\left|x_{3}-x_{03}\right|<R\right\}, \quad \mathcal{C}(R)=\mathcal{C}(0, R), \quad \mathcal{C}=\mathcal{C}(1) ; \\
\left.z=(x, t), \quad z_{0}=\left(x_{0}, t_{0}\right), \quad Q\left(z_{0}, R\right)=\mathcal{C}\left(x_{0}, R\right) \times\right] t_{0}-R^{2}, t_{0}[, \\
Q(R)=Q(0, R), \quad Q=Q(1) .
\end{gathered}
$$

In local analysis, the most reasonable object to study is so-called suitable weak solutions, introduced by Caffarelli-Kohn-Nirenberg in their celebrated paper [1]. We are going to use a slightly simpler definition of F.-H. Lin in 10 . 
Definition 1.1 The pair $v$ and $p$ is called a suitable weak solutions to the Navier-Stokes equations in $Q$ if the following conditions are satisfied:

$$
v \in L_{2, \infty}(Q) \cap W_{2}^{1,0}(Q), \quad p \in L_{\frac{3}{2}}(Q) ;
$$

$v$ andp satisfy the Navier-Stokes equtions in the sense of distributions; for a.a. $t \in]-1,0[$, the local energy inequality

$$
\begin{aligned}
\int_{\mathcal{C}} \varphi(x, t)|v(x, t)|^{2} d x & +2 \int_{-1}^{t} \int_{\mathcal{C}} \varphi|\nabla v|^{2} d x d t^{\prime} \leq \int_{-1}^{t} \int_{\mathcal{C}}\left\{|v|^{2}\left(\Delta \varphi+\partial_{t} v\right)+\right. \\
& \left.+v \cdot \nabla \varphi\left(|v|^{2}+2 p\right)\right\} d x d t^{\prime}
\end{aligned}
$$

holds for all non-negative cut-off functions $\varphi \in C_{0}^{\infty}\left(\mathbb{R}^{3} \times \mathbb{R}\right)$ vanishing in a neighborhood of the parabolic boundary of $Q$.

For discussions of the above definition, we refer the reader to papers [8] and [17.

Our main result is

Theorem 1.2 Let $v$ and $p$ be an axially symmetric suitable weak solution to the Navier-Stokes equations in $Q$. Assume that

$$
\mathcal{A}_{0}=\operatorname{ess} \sup _{-1 \leq t \leq 0} \int_{\mathcal{C}} \frac{1}{\varrho}|v(x, t)|^{2} d x<+\infty .
$$

Then the point $(x, t)=(0,0)$ is a regular point of $v$, i.e., there exists $r \in] 0,1]$ such that $v$ is Hölder continuous in the closure of the cylinder $Q(r)$.

By $c$, we shall denote all generic constants that may vary from one bound to others.

Our paper is organized as follows. In the second section, we discuss known inequalities of the local regularity theory and prove some useful facts about suitable weak solutions. The proof of the main result is started in the third section with scaling and blow up of our solution at a singular point. We also discuss properties of the blow up velocity and the blow up pressure in this section. In the fourth section, we prove some additional differential properties of axially symmetric suitable weak solutions. They are needed to 
establish a decay of the blow up velocity at infinity. Finally, we end up with the proof of the main theorem in the fifth section. Here, with the help of backward uniqueness results for the heat operator with variable lower order terms, we show that in fact our blow up velocity is trivial.

Acknowledgement The work is supported by the Agreement on cooperation between Polish and Russian Academies of Sciences signed in Warsaw, Dec. 27, 2002. The first author is supported by the Alexander von Humboldt Foundation and by the RFFI grant 05-01-00941-a.

\section{Preliminaries}

In what follows, we are going to make use of the following scaling invariant functionals:

$$
\begin{gathered}
A\left(z_{0}, r ; v\right)=\mathrm{ess} \sup _{t_{0}-r^{2}<t<t_{0}} \frac{1}{r} \int_{\mathcal{C}\left(x_{0}, r\right)}|v(x, t)|^{2} d x, \quad C\left(z_{0}, r ; v\right)=\frac{1}{r^{2}} \int_{Q\left(z_{0}, r\right)}|v|^{3} d z, \\
E\left(z_{0}, r ; v\right)=\frac{1}{r} \int_{Q\left(z_{0}, r\right)}|\nabla v|^{2} d z, \quad D\left(z_{0}, r ; p\right)=\frac{1}{r^{2}} \int_{Q\left(z_{0}, r\right)}|p|^{\frac{3}{2}} d z .
\end{gathered}
$$

First, let us recall that, by the Navier-Stokes equations scaling,

$$
v^{\lambda}(x, t)=\lambda v\left(\lambda x, \lambda^{2} t\right), \quad p^{\lambda}(x, t)=\lambda^{2} p\left(\lambda x, \lambda^{2} t\right),
$$

we may define suitable weak solutions to the Navier-Stokes equations in $Q\left(z_{0}, R\right)$. So, if $v$ and $p$ form a suitable weak solution to the Navier-Stokes equations in $Q\left(z_{0}, R\right)$, then, for appropriate choice of the cut-off function in the local energy inequality, we can reduce it to the following invariant form

$$
\begin{gathered}
A\left(z_{0}, R / 2 ; v\right)+E\left(z_{0}, R / 2 ; v\right) \leq c\left(C^{\frac{2}{3}}\left(z_{0}, R ; v\right)+\right. \\
\left.+C\left(z_{0}, R ; v\right)+D\left(z_{0}, R ; p\right)\right) .
\end{gathered}
$$

We also need the so-called decay estimate for pressure

$$
D\left(z_{0}, r ; p\right) \leq c\left[\frac{r}{r_{1}} D\left(z_{0}, r_{1} ; p\right)+\left(\frac{r_{1}}{r}\right)^{2} C\left(z_{0}, r_{1} ; v\right)\right],
$$

which is valid for all $0<r \leq r_{1} \leq R$. The proof of the latter estimate is given in [14]. Repeating arguments of Lemma 1.8 in [18], we can prove 
Lemma 2.1 Let $v$ and $p$ be a suitable weak solution to the Navier-Stokes equations in $Q$ and let

$$
A_{0}=\sup _{0<r<1} A(0, r ; v)<+\infty .
$$

Then, for any $r \in] 0,1 / 2[$, we have

$$
\begin{gathered}
C^{\frac{4}{3}}(0, r ; v)+D(0, r ; p)+E(0, r ; v) \leq c\left(\left(A_{0}+1\right) r^{\frac{1}{2}}(D(0,1 ; p)+\right. \\
\left.+E(0,1 ; v))+A_{0}^{4}+A_{0}^{2}+A_{0}\right)
\end{gathered}
$$

Lemma 2.1, together with the invariance of our functionals under the Navier-Stokes equations scaling and under the shift in the direction of $x_{3}$, gives us:

Lemma 2.2 Under the conditions of Theorem 1.2, we have

$$
A\left(z_{0}, r ; v\right)+C\left(z_{0}, r ; v\right)+D\left(z_{0}, r ; p\right)+E\left(z_{0}, r ; v\right) \leq \mathcal{A}<+\infty
$$

for all $z_{0}=\left(x_{0}, 0\right), x_{0}=(0, b),|b| \leq 1 / 4$, and for all $0<r \leq 1 / 4$, where $\mathcal{A}$ depends on $D(0,1 ; p), E(0,1 ; v)$, and $\mathcal{A}_{0}$ only.

We say that the pair $v$ and $p$ is a suitable weak solution to the NavierStokes equations in the space-time cylinder $\Omega \times] T_{1}, T_{2}\left[\right.$, if, for any $z_{0}=\left(x_{0}, t_{0}\right)$ with $x_{0} \in \Omega$ and $T_{1}<t \leq T_{2}$, the pair $v$ and $p$ is a suitable weak solution to the Navier-Stokes equations in $Q\left(z_{0}, R\right)$ for some $R>0$.

Next, let us introduce the family of sets

$$
\mathcal{P}\left(R_{1}, R_{2} ; a\right)=\left\{x \in \mathbb{R}^{3} \| R_{1}<\left|x^{\prime}\right|<R_{2},\left|x_{3}\right|<a\right\} .
$$

Now, we would like to formulate and prove the following statement.

Lemma 2.3 Let $v$ and $p$ be a suitable weak solution to the Navier-Stokes equations in the set $\widehat{Q}=\mathcal{P}(3 / 4,9 / 4 ; 3 / 2) \times]-(3 / 2)^{2}, 0[$. Assume that

$$
\int_{\widehat{Q}}|v(z)|^{6} d z \leq m<+\infty .
$$


Then, there exists a function $\Phi_{0}: \mathbb{R}_{+} \times \mathbb{R}_{+} \rightarrow \mathbb{R}_{+}$, nondecreasing in each variables, such that

$$
|v(z)|+|\nabla v(z)| \leq \Phi_{0}\left(m, \mathcal{A}_{*}\right)<+\infty,
$$

for any $z \in \mathcal{P}(1,2 ; 1) \times]-1,0[$. Here,

$$
\mathcal{A}_{*}=\int_{\widehat{Q}}|p(z)|^{\frac{3}{2}} d z=\int_{-(3 / 2)^{2}}^{0} d t \int_{\mathcal{P}(3 / 4,9 / 4 ; 3 / 2)}|p|^{\frac{3}{2}} d x .
$$

Proof First, we remark $Q\left(z_{0}, 1 / 4\right) \subset \widehat{Q}$ for any $\left.z_{0} \in \mathcal{P}(1,2 ; 1) \times\right]-1,0[$. It follows from (2.2), Hölder's inequality, and (2.6) that

$$
D\left(z_{0}, r ; p\right) \leq c\left[\frac{r}{r_{1}} D\left(z_{0}, r_{1} ; p\right)+\left(\frac{r_{1}}{r}\right)^{2} m^{\frac{1}{2}} r_{1}^{\frac{1}{2}}\right],
$$

which is valid for all $0<r \leq r_{1} \leq 1 / 4$. For $\left.\tau \in\right] 0,1$, let us take $r=\tau^{k+1} / 4$ and $r_{1}=\tau^{k} / 4$ in (2.8) and find

$$
D\left(z_{0}, \tau^{k+1} / 4 ; p\right) \leq c \tau\left[D\left(z_{0}, \tau^{k} / 4 ; p\right)+m^{\frac{1}{2}} \tau^{-3} \tau^{\frac{k}{2}}\right]
$$

for all non-negative integer numbers $k$. We can choose $\tau \in] 0,1[$ so small to provide

$$
c \tau^{\frac{3}{4}} \leq 1
$$

and conclude

$$
D\left(z_{0}, \tau^{k+1} / 4 ; p\right) \leq \tau^{\frac{1}{4}}\left[D\left(z_{0}, \tau^{k} / 4 ; p\right)+m^{\frac{1}{2}} \tau^{-3} \tau^{\frac{k}{2}}\right]
$$

for all non-negative integer numbers $k$. The latter inequality may be easily iterated. As a result, we have

$$
D\left(z_{0}, \tau^{k+1} / 4 ; p\right) \leq \tau^{\frac{k+1}{4}}\left[D\left(z_{0}, 1 / 4 ; p\right)+m^{\frac{1}{2}} \tau^{-3} \sum_{i=0}^{k} \tau^{\frac{i}{4}}\right]
$$

for all non-negative integer numbers $k$. So,

$$
C\left(z_{0}, \tau^{k+1} / 4 ; v\right)+D\left(z_{0}, \tau^{k+1} / 4 ; p\right) \leq c m^{\frac{1}{2}} \tau^{\frac{k+1}{2}}+\tau^{\frac{k+1}{4}}\left[D\left(z_{0}, 1 / 4 ; p\right)\right.
$$




$$
\begin{gathered}
\left.+m^{\frac{1}{2}} \tau^{-3}\left(1-\tau^{\frac{1}{4}}\right)^{-1}\right] \\
\leq c\left[m^{\frac{1}{2}} \tau^{\frac{k+1}{2}}+\tau^{\frac{k+1}{4}}\left(\mathcal{A}_{*}+m^{\frac{1}{2}} \tau^{-3}\left(1-\tau^{\frac{1}{4}}\right)^{-1}\right)\right]
\end{gathered}
$$

for all non-negative integer numbers $k$. Given $\varepsilon>0$, we can find an integer number $k_{0}$ so that

$$
c\left[m^{\frac{1}{2}} \tau^{\frac{k_{0}+1}{2}}+\tau^{\frac{k_{0}+1}{4}}\left(\mathcal{A}_{*}+m^{\frac{1}{2}} \tau^{-3}\left(1-\tau^{\frac{1}{4}}\right)^{-1}\right)\right] \leq \varepsilon .
$$

But according to the so-called $\varepsilon$-regularity theory, see, for example, [8], [4], and [17, the latter implies two bounds:

$$
\left|v\left(z_{0}\right)\right| \leq \frac{c}{r_{0}} \quad \text { and } \quad\left|\nabla v\left(z_{0}\right)\right| \leq \frac{c}{r_{0}^{2}}
$$

where $r_{0}=\tau^{\left(k_{0}+1\right)} / 4$. Lemma 2.3 is proved.

The last preliminary statement is as follows.

Lemma 2.4 Assume that all conditions of Theorem 1.2 hold. Then

$$
\int_{\mathcal{C}} \frac{1}{\varrho}|v(x, t)|^{2} d x \leq \mathcal{A}_{0}
$$

for all $t \in]-1,0[$.

Proof It easy to derive the following estimate

$$
\int_{Q} \partial_{t} v \cdot w d z \leq \mathcal{A}_{1}\left(\int_{Q}|\nabla w|^{3} d z\right)^{\frac{1}{3}}
$$

for any $C_{0}^{\infty}(Q)$. Here, a constant $\mathcal{A}_{1}$ depends on $C(0,1 ; v), E(0,1 ; v)$, and $D(0,1 ; p)$ only. So, $v$ has the first derivative in to $t$ in the space

$$
L_{\frac{3}{2}}\left(-1,0 ; W_{\frac{3}{2}}^{-1}(\mathcal{C})\right) .
$$

In turn, the latter, together with boundedness of the energy, implies weak continuity in time in the following sense: the function

$$
t \rightarrow \int_{\mathcal{C}} v(x, t) \cdot w(x) d x
$$


is continuous on $[-1,0]$ for any $w \in L_{2}(\mathcal{C})$. Now, the statement of the lemma follows from the weak lower semicontinuity of the functional

$$
w \in L_{2}(\mathcal{C}) \rightarrow \int_{\mathcal{C}} \frac{1}{\varrho}|w(x)|^{2} d x .
$$

Lemma 2.4 is proved.

\section{Scaling and Blow Up}

Here, we are starting with the proof of Theorem 1.2. Assume that the statement of this theorem is false. Then, according to the local regularity theory for the Navier-Stokes equations, there exist an absolute positive constant $\varepsilon$ and a sequence $\left\{R_{k}\right\}_{k=1}^{\infty}$ such that $R_{k} \rightarrow 0$ as $k \rightarrow+\infty$ and

$$
\frac{1}{R_{k}^{2}} \int_{Q\left(R_{k}\right)}|v|^{3} d z \geq \varepsilon>0
$$

for all $k \in \mathbb{N}$.

Next, we scale $v$ and $p$ in the following way

$$
u^{k}(y, s)=R_{k} v\left(R_{k} y, R_{k}^{2} s\right), \quad q^{k}(y, s)=R_{k}^{2} p\left(R_{k} y, R_{k}^{2} s\right),
$$

where $e=(y, s) \in Q\left(1 / R_{k}\right)$. Functions $u^{k}$ and $q^{k}$ are extended by zero to the whole space-time $\mathbb{R}^{3} \times \mathbb{R}$.

Now let us fix numbers $a$ and $b$ in $\mathbb{R}$ so that $a>0$. Let

$$
x_{k}^{b}=\left(0, b R_{k}\right), \quad y^{b}=(0, b), \quad z_{k}^{b}=\left(x_{k}^{b}, 0\right), \quad e^{b}=\left(y^{b}, 0\right) .
$$

Obviously, for sufficiently large $k$,

$$
|b| R_{k}<1 / 4, \quad a R_{k}<1 / 4
$$

by Lemma 2.2 , the following estimates are valid:

$$
\begin{aligned}
& C\left(z_{k}^{b}, a R_{k} ; v\right)=C\left(e^{b}, a ; u^{k}\right) \leq \mathcal{A}, \\
& E\left(z_{k}^{b}, a R_{k} ; v\right)=E\left(e^{b}, a ; u^{k}\right) \leq \mathcal{A}, \\
& A\left(z_{k}^{b}, a R_{k} ; v\right)=A\left(e^{b}, a ; u^{k}\right) \leq \mathcal{A},
\end{aligned}
$$




$$
D\left(z_{k}^{b}, a R_{k} ; p\right)=D\left(e^{b}, a ; q^{k}\right) \leq \mathcal{A}
$$

for all $k \geq k_{0}(a, b)$.

First, let $b$ be equal to zero. In this particular case, we can produce three estimates. The first of them is well known in the Navier-Stokes theory and it is but a consequence of multiplicative inequalities

$$
\frac{1}{a^{\frac{5}{2}}} \int_{Q(a)}\left|u^{k}\right|^{\frac{10}{3}} d e \leq c(\mathcal{A})
$$

The second estimate follows from the Navier-Stokes equations, written for $u^{k}$ and $q^{k}$ in the weak form, and from (3.2):

$$
\int_{Q(a)} \partial_{t} u^{k} \cdot w d e \leq c(a, \mathcal{A})\left(\int_{Q(a)}|\nabla w|^{3} d e\right)^{\frac{1}{3}}
$$

for all $w \in C_{0}^{\infty}(Q(a))$. Hence,

$$
\partial_{t} u^{k} \text { is bounded in } L_{\frac{3}{2}}\left(-a^{2}, 0 ; W_{\frac{3}{2}}^{-1}(\mathcal{C}(a))\right) .
$$

The third estimate is coming from our main condition (1.3) and has the form

$$
\operatorname{ess} \sup _{-\left(a R_{k}\right)^{2} \leq t \leq 0} \int_{\mathcal{C}\left(a R_{k}\right)} \frac{|v(x, t)|^{2}}{\left|x^{\prime}\right|} d x=\operatorname{ess} \sup _{-a^{2} \leq s \leq 0} \int_{\mathcal{C}(a)} \frac{\left|u^{k}(y, t)\right|^{2}}{\left|y^{\prime}\right|} d y \leq \mathcal{A}_{0}
$$

Now, making use of the diagonal process for extending space-time cylinders $Q(a)$ and known compactness arguments, we can select subsequences (still denoted by $u^{k}$ and $q^{k}$ ) such that, for each $a>0$,

$$
\begin{array}{cc}
u^{k} \rightarrow u & \text { in } W_{2}^{1,0}(Q(a)), \\
u^{k} \stackrel{\star}{\rightarrow} u & \text { in } L_{2, \infty}(Q(a)), \\
u^{k} \rightarrow u & \text { in } L_{3}(Q(a)), \\
q^{k} \rightarrow q & \text { in } L_{\frac{3}{2}}(Q(a)) .
\end{array}
$$

The aim of our further considerations is to describe properties of limit functions $u$ and $q$ called the blow up velocity and blow up pressure, respectively. They are defined on $\mathbb{R}^{3} \times \mathbb{R}_{-}$, where $\mathbb{R}_{-}=\{s \in \mathbb{R} \| s \leq 0\}$. For each $a>0$, 
the pair $u$ and $q$ is a suitable weak solution to the Navier-Stokes equations in $Q(a)$. From (3.2) and (3.6), it follows that the limit functions obey the inequalities:

$$
\begin{gathered}
C\left(e^{b}, a ; u\right) \leq \mathcal{A}, \\
A\left(e^{b}, a ; u\right) \leq \mathcal{A}, \\
E\left(e^{b}, a ; u\right) \leq \mathcal{A}, \\
D\left(e^{b}, a ; q\right) \leq \mathcal{A}
\end{gathered}
$$

for all $b \in \mathbb{R}$ and for all $0<a \in \mathbb{R}$. Moreover, we can derive from (3.6), (3.5), and (3.1) two additional estimates:

$$
\operatorname{ess} \sup _{-\infty<s \leq 0} \int_{\mathbb{R}^{3}} \frac{|u(y, t)|^{2}}{\left|y^{\prime}\right|} d y \leq \mathcal{A}_{0}
$$

and

$$
\frac{1}{R_{k}^{2}} \int_{Q\left(R_{k}\right)}|v|^{3} d z=\int_{Q}\left|u^{k}\right|^{3} d e \rightarrow \int_{Q}|u|^{3} d e \geq \varepsilon .
$$

According to (3.9), the blow up velocity $u$ is a non-trivial solution to the Navier-Stokes equations in $\mathbb{R}^{3} \times \mathbb{R}_{-}$. But we are going to show that in fact $u \equiv 0$. This would contradict with (3.9) and prove Theorem 1.2 .

Obviously, the blow up velocity field $u$ is axially symmetric and, by Caffarerrli-Kohn-Nirenberg type results, all point $y^{\prime} \neq 0$ are regular which make it possible to conclude that all spatial derivatives of $u$ are Hölder continuous in a vicinity of each point with $y^{\prime} \neq 0$.

We can also make use of the local regularity theory for Stokes system, see [16] and [17]. According to it and by known multiplicative inequality, we have

$$
\begin{gathered}
\left\|\partial_{t} u^{k}\right\|_{L_{\frac{9}{8}, \frac{3}{2}}(Q(a / 2))}+\left\|\nabla^{2} u^{k}\right\|_{L_{\frac{9}{8}, \frac{3}{2}}(Q(a / 2))}+\left\|\nabla q^{k}\right\|_{L_{\frac{9}{8}, \frac{3}{2}}(Q(a / 2))} \leq \\
\leq c(a)\left[\left\|u^{k} \cdot \nabla u^{k}\right\|_{L_{\frac{9}{8}, \frac{3}{2}}(Q(a))}+\left\|u^{k}\right\|_{W_{2}^{1,0}(Q(a))}+\left\|q^{k}\right\|_{L_{\frac{3}{2}}(Q(a))}\right] \leq \\
\leq c(a)\left[\left\|u^{k}\right\|_{L_{2, \infty}(Q(a))}^{\frac{2}{3}}\left\|u^{k}\right\|_{W_{2}^{1,0}(Q(a))}^{\frac{1}{3}}+\ldots\right] \leq c(a, \mathcal{A}) .
\end{gathered}
$$

The latter estimate shows that we can select a subsequence (still denoted by $u^{k}$ ) such that, for any $a>1$,

$$
u^{k} \rightarrow u \quad \text { in } C\left([-1,0] ; L_{\frac{9}{8}}(\mathcal{C}(a))\right) .
$$


(3.10) can be exploited in the following way. For any fixed positive numbers $r_{1}, r_{2}$, and $h$, we have

$$
\begin{gathered}
\left(\int_{\mathcal{P}\left(r_{1}, r_{2} ; h\right)}|u(y, 0)|^{\frac{9}{8}} d y\right)^{\frac{8}{9}} \leq\left(\int_{\mathcal{P}\left(r_{1}, r_{2} ; h\right)}\left|u^{k}(y, 0)-u(y, 0)\right|^{\frac{9}{8}} d y\right)^{\frac{8}{9}}+ \\
+\left(\int_{\mathcal{P}\left(r_{1}, r_{2} ; h\right)}\left|u^{k}(y, 0)\right|^{\frac{9}{8}} d y\right)^{\frac{8}{9}}=\alpha_{k}+\beta_{k} .
\end{gathered}
$$

By (3.10),

$$
\alpha_{k} \rightarrow 0
$$

as $k \rightarrow+\infty$. To evaluate $\beta_{k}$, we make use of the inverse scaling and Hölder's inequality

$$
\begin{gathered}
\beta_{k}=\left(R_{k}^{-\frac{15}{8}} \int_{\mathcal{P}\left(R_{k} r_{1}, R_{k} r_{2} ; R_{k} h\right)}|v(x, 0)|^{\frac{9}{8}} d x\right)^{\frac{8}{9}} \leq \\
\leq c\left(r_{1}, r_{2}, h\right)\left(\frac{1}{R_{k}} \int_{\mathcal{P}\left(R_{k} r_{1}, R_{k} r_{2} ; R_{k} h\right)}|v(x, 0)|^{2} d x\right)^{\frac{1}{2}} \leq \\
\leq c\left(r_{1}, r_{2}, h\right)\left(\int_{\mathcal{P}\left(R_{k} r_{1}, R_{k} r_{2} ; R_{k} h\right)} \frac{|v(x, 0)|^{2}}{\left|x^{\prime}\right|} d x\right)^{\frac{1}{2}} .
\end{gathered}
$$

Now, it remains to apply Lemma 2.4 at $t=0$ and absolute continuity of Lebesgue's integral and conclude that

$$
\beta_{k} \rightarrow 0
$$

as $k \rightarrow+\infty$. This implies the identity

$$
\int_{\mathcal{P}\left(r_{1}, r_{2} ; h\right)}|u(y, 0)|^{\frac{9}{8}} d y=0
$$

for all positive numbers $r_{1}, r_{2}$, and $h$. So, we can state that

$$
u(\cdot, 0)=0 \quad \text { in } \quad \mathbb{R}^{3} .
$$




\section{Estimates of Axially Symmetric Solutions}

The main result of this section is going to be as follows.

Proposition 4.1 Let $V$ and $P$ be a sufficiently smooth axially symmetric solution to the Navier-Stokes equations in $\left.\widetilde{Q}=\widetilde{\mathcal{P}}_{\times}\right]-2^{2}, 0[$, where $\widetilde{\mathcal{P}}=$ $\mathcal{P}(1 / 4,3 ; 2)$. Then, there exists a non-decreasing function $\Phi: \mathbb{R}_{+} \rightarrow \mathbb{R}_{+}$such that

$$
\sup _{z \in \mathcal{P}(1,2 ; 1) \times]-1,0[}(|V(z)|+|\nabla V(z)|) \leq \Phi\left(\mathcal{A}_{2}\right),
$$

where

$$
\mathcal{A}_{2}=\sup _{-2^{2}<t<0} \int_{\widetilde{\mathcal{P}}}|V(x, t)|^{2} d x+\int_{\widetilde{Q}}\left(|\nabla V|^{2}+|V|^{3}+|P|^{\frac{3}{2}}\right) d z .
$$

To prove the above proposition, we need

Lemma 4.2 Under assumptions of Proposition 4.1, there exists a function $\Phi_{1}: \mathbb{R}_{+} \times \mathbb{R}_{+} \rightarrow \mathbb{R}_{+}$, non-decreasing in each variable, such that

$$
\sup _{-(7 / 4)^{2}<t<0} \int_{\widetilde{\mathcal{P}}_{1}}\left|V^{a}(x, t)\right|^{q} d x \leq \Phi_{1}\left(q, \mathcal{A}_{2}\right), \quad 1 \leq q+\infty .
$$

Here, $V^{a}=\left(V_{\varrho}, V_{3}\right),\left|V^{a}\right|=\sqrt{\left|V_{\varrho}\right|^{2}+\left|V_{3}\right|^{2}}, \widetilde{\mathcal{P}}_{1}=\mathcal{P}(5 / 16,11 / 4 ; 7 / 4)$, and $\left.\widetilde{Q}_{1}=\widetilde{\mathcal{P}}_{1} \times\right]-(7 / 4)^{2}, 0[$.

Proof Let us denote by $\omega$ the vorticity of $v$, i.e., $\omega=\nabla \wedge v$. For $\chi=\omega_{\varphi}$, $V_{\varrho}$, and $V_{3}$, we have the following identities:

$$
\begin{gathered}
V_{\varrho, \varrho}+V_{3,3}=-\frac{1}{\varrho} V_{\varrho}, \\
V_{\varrho, 3}-V_{3, \varrho}=\chi, \\
\partial_{t} \chi+V_{\varrho} \chi_{, \varrho}+V_{3} \chi_{, 3}-\frac{1}{\varrho} \chi V_{\varrho}-\left(\chi_{, \varrho \varrho}+\chi_{, 33}+\frac{1}{\varrho} \chi_{, \varrho}-\frac{1}{\varrho^{2}} \chi\right)= \\
=\frac{2}{\varrho} V_{\varphi} V_{\varphi, 3},
\end{gathered}
$$


where we have used the notion

$$
f_{, \varrho}=\frac{\partial f}{\partial \varrho}, \quad f_{, 3}=\frac{\partial f}{\partial x_{3}} .
$$

Next, we let $\tilde{\chi}=\chi \psi, \widetilde{V}=V^{a} \psi, \widetilde{V}_{\varrho}=V_{\varrho} \psi$, and $\widetilde{V}_{3}=V_{3} \psi$, where a nonnegative smooth and axially symmetric cut-off function $\psi$ vanishes in a neighborhood of the parabolic boundary of $\widetilde{Q}$ and is equal to 1 in $\widetilde{Q}_{1}$. For $\widetilde{\chi}, \widetilde{V}_{\varrho}$, and $\widetilde{V}_{3}$, we have

$$
\begin{gathered}
\tilde{V}_{\varrho, \varrho}+\widetilde{V}_{3,3}=-\frac{1}{\varrho} \widetilde{V}_{\varrho}+V_{\varrho} \psi_{, \varrho}+V_{3} \psi_{, 3}, \\
\widetilde{V}_{\varrho, 3}-\widetilde{V}_{3, \varrho}=\tilde{\chi}+V_{\varrho} \psi_{, 3}-V_{3} \psi_{, \varrho}, \\
\partial_{t} \tilde{\chi}+V_{\varrho} \widetilde{\chi}_{, \varrho}+V_{3} \widetilde{\chi}_{, 3}-\frac{1}{\varrho} V_{\varrho} \tilde{\chi}-\left(\tilde{\chi}_{, \varrho \varrho}+\widetilde{\chi}_{, 33}+\frac{1}{\varrho} \widetilde{\chi}_{, \varrho}-\frac{1}{\varrho^{2}} \tilde{\chi}\right)= \\
=J_{1}+J_{2}+J_{3},
\end{gathered}
$$

where

$$
\begin{gathered}
J_{1}=\frac{2}{\varrho} V_{\varphi} V_{\varphi, 3} \psi \\
J_{2}=\chi\left(\partial_{t} \psi-\psi_{, \varrho \varrho}-\psi_{, 33}-\frac{1}{\varrho} \psi_{, \varrho}\right)-2\left(\chi_{, \varrho} \psi_{, \varrho}+\chi_{, 3} \psi_{, 3}\right), \\
J_{3}=\chi\left(V_{\varrho} \psi_{, \varrho}+V_{3} \psi_{, 3}\right) .
\end{gathered}
$$
$\widetilde{\mathcal{P}}$

Now, we multiply (4.8) by $\tilde{\chi} \varrho^{-2}$ and integrate the product by parts over

$$
\begin{gathered}
\frac{1}{2} \partial_{t} \int_{\widetilde{\mathcal{P}}}\left|\frac{\tilde{\chi}}{\varrho}\right|^{2} d x+\int_{\widetilde{\mathcal{P}}}\left(\left|\left(\frac{\tilde{\chi}}{\varrho}\right)_{, \varrho}\right|^{2}+\left|\left(\frac{\widetilde{\chi}}{\varrho}\right)_{, 3}\right|^{2}\right) d x= \\
=\int_{\widetilde{\mathcal{P}}} J_{1} \frac{\widetilde{\chi}}{\varrho^{2}} d x+\int_{\widetilde{\mathcal{P}}} J_{2} \frac{\widetilde{\chi}}{\varrho^{2}} d x+\int_{\widetilde{\mathcal{P}}} J_{3} \frac{\widetilde{\chi}}{\varrho^{2}} d x .
\end{gathered}
$$

Our aim is to evaluate the right hand side of (4.9). We start with the first term there:

$$
\int_{\widetilde{\mathcal{P}}} J_{1} \frac{\tilde{\chi}}{\varrho^{2}} d x=-\int_{\widetilde{\mathcal{P}}} \frac{V_{\varphi}^{2}}{\varrho^{2}}\left(\frac{\widetilde{\chi}}{\varrho}\right)_{, 3} \psi d x-\int_{\widetilde{\mathcal{P}}} \frac{V_{\varphi}^{2}}{\varrho^{2}} \frac{\widetilde{\chi}}{\varrho} \psi_{, 3} d x \leq
$$




$$
\leq c\left(\int_{\widetilde{\mathcal{P}}} \frac{\left|V_{\varphi}\right|^{4}}{\varrho^{4}} d x\right)^{\frac{1}{2}}\left(\int_{\widetilde{\mathcal{P}}}\left|\left(\frac{\widetilde{\chi}}{\varrho}\right)_{, 3}\right|^{2} d x+\int_{\widetilde{\mathcal{P}}}\left|\frac{\widetilde{\chi}}{\varrho}\right|^{2} d x\right)^{\frac{1}{2}},
$$

where the notion $\nabla_{a} f=\left(f_{,}, f_{, 3}\right)$ has been used. To estimate the first multiplier of the right hand of the latter inequality, we are going to exploit two-dimensional feature of our axially symmetric problem in the following way. So, by Ladyzhenskaya's inequality,

$$
\begin{gathered}
\int_{\widetilde{\mathcal{P}}}\left|V_{\varphi}\right|^{4} d x \leq c \int_{-2}^{2} \int_{1 / 4}^{3}\left|V_{\varphi}\right|^{4} d \varrho d x_{3} \leq \\
\leq c \int_{-2}^{2} \int_{1 / 4}^{3}\left|V_{\varphi}\right|^{2} d \varrho d x_{3} \int_{-2}^{2} \int_{1 / 4}^{3}\left(\left|V_{\varphi}\right|^{2}+\left|\nabla_{a} V_{\varphi}\right|^{2}\right) d \varrho d x_{3} \leq \\
\leq c \int_{\widetilde{\mathcal{P}}}|V|^{2} d x \int_{\widetilde{\mathcal{P}}}\left(|V|^{2}+|\nabla V|^{2}\right) d x \leq c \mathcal{A}_{2} \int_{\widetilde{\mathcal{P}}}\left(|V|^{2}+|\nabla V|^{2}\right) d x .
\end{gathered}
$$

Thus, we find the first estimate:

$$
\begin{gathered}
\int_{\widetilde{\mathcal{P}}} J_{1} \frac{\tilde{\chi}}{\varrho^{2}} d x \leq c \mathcal{A}_{2}^{\frac{1}{2}}\left(\int_{\widetilde{\mathcal{P}}}\left(|V|^{2}+|\nabla V|^{2}\right) d x\right)^{\frac{1}{2}} \times \\
\times\left(\int_{\widetilde{\mathcal{P}}}\left|\nabla_{a}\left(\frac{\widetilde{\chi}}{\varrho}\right)\right|^{2} d x+\int_{\widetilde{\mathcal{P}}}|\underline{\widetilde{\chi}}|^{2} d x\right)^{\frac{1}{2}} .
\end{gathered}
$$

For the second term, we have

$$
\int_{\widetilde{\mathcal{P}}} J_{2} \frac{\widetilde{\chi}}{\varrho^{2}} d x \leq c \int_{\widetilde{\mathcal{P}}}|\chi|^{2} d x
$$

The third term is estimated in slightly different way

$$
\begin{gathered}
\int_{\widetilde{\mathcal{P}}} J_{3} \frac{\tilde{\chi}}{\varrho^{2}} d x=\int_{\widetilde{\mathcal{P}}} \frac{\chi \widetilde{\chi}}{\varrho^{2}}\left(V_{\varrho} \psi_{, \varrho}+V_{3} \psi_{, 3}\right) d x \leq \\
\leq c\left(\int_{\widetilde{\mathcal{P}}}|\chi|^{2} d x\right)^{\frac{1}{2}}\left(\int_{\widetilde{\mathcal{P}}}\left|V^{a} \cdot \nabla_{a} \psi\right|^{2}\left|\frac{\tilde{\chi}}{\varrho}\right|^{2} d x\right)^{\frac{1}{2}} \leq
\end{gathered}
$$




$$
\leq c \int_{\widetilde{\mathcal{P}}}|\chi|^{2} d x+c\left(\int_{\widetilde{\mathcal{P}}}\left|V^{a} \cdot \nabla_{a} \psi\right|^{4} d x\right)^{\frac{1}{2}}\left(\int_{\widetilde{\mathcal{P}}}\left|\frac{\tilde{\chi}}{\varrho}\right|^{4} d x\right)^{\frac{1}{2}},
$$

where we let $V^{a} \cdot \nabla_{a} \psi=V_{\varrho} \psi_{, \varrho}+V_{3} \psi_{, 3}$. To estimate the last term on the right hand side of the latter relation, we exploit Ladyzhenskaya's inequality once more. So, we have

$$
\begin{gathered}
\int_{\widetilde{\mathcal{P}}}\left|\frac{\tilde{\chi}}{\varrho}\right|^{4} d x \leq c \int_{-2}^{2} \int_{1 / 4}^{3}\left|\frac{\tilde{\chi}}{\varrho}\right|^{4} d \varrho d x_{3} \leq \\
\leq c \int_{-2}^{2} \int_{1 / 4}^{3}\left|\nabla_{a}\left(\frac{\tilde{\chi}}{\varrho}\right)\right|^{2} d \varrho d x_{3} \int_{-2}^{2} \int_{1 / 4}^{3}\left|\frac{\tilde{\chi}}{\varrho}\right|^{2} d \varrho d x_{3} \leq \\
\leq c \int_{\widetilde{\mathcal{P}}}\left|\nabla_{a}\left(\frac{\tilde{\chi}}{\varrho}\right)\right|^{2} d x \int_{\widetilde{\mathcal{P}}}\left|\frac{\tilde{\chi}}{\varrho}\right|^{2} d x
\end{gathered}
$$

and, in the same way,

$$
\int_{\widetilde{\mathcal{P}}}\left|V^{a} \cdot \nabla_{a} \psi\right|^{4} d x \leq c \int_{\widetilde{\mathcal{P}}}\left|\nabla_{a}\left(V^{a} \cdot \nabla_{a} \psi\right)\right|^{2} d x \int_{\widetilde{\mathcal{P}}}\left|V^{a} \cdot \nabla_{a} \psi\right|^{2} d x .
$$

As a result, we find

$$
\begin{gathered}
\int_{\widetilde{\mathcal{P}}} J_{3} \frac{\widetilde{\chi}}{\varrho^{2}} d x \leq c \int_{\widetilde{\mathcal{P}}}|\chi|^{2} d x+c\left(\int_{\widetilde{\mathcal{P}}}\left|V_{a}\right|^{2} d x+\int_{\widetilde{\mathcal{P}}}\left|\nabla_{a} V^{a}\right|^{2} d x\right)^{\frac{1}{2}} \times \\
\times\left(\int_{\widetilde{\mathcal{P}}}\left|V_{a}\right|^{2} d x\right)^{\frac{1}{2}}\left(\int_{\widetilde{\mathcal{P}}}\left|\nabla_{a}\left(\frac{\tilde{\chi}}{\varrho}\right)\right|^{2} d x\right)^{\frac{1}{2}}\left(\int_{\widetilde{\mathcal{P}}}\left|\frac{\widetilde{\chi}}{\varrho}\right|^{2} d x\right)^{\frac{1}{2}} \leq \\
\leq c \int_{\widetilde{\mathcal{P}}}|\nabla V|^{2} d x+c\left(\mathcal{A}_{2}+\mathcal{A}_{2}^{\frac{1}{2}}\left(\int_{\widetilde{\mathcal{P}}}|\nabla V|^{2} d x\right)^{\frac{1}{2}}\right) \times \\
\times\left(\int_{\widetilde{\mathcal{P}}}\left|\nabla_{a}\left(\frac{\tilde{\chi}}{\varrho}\right)\right|^{2} d x\right)^{\frac{1}{2}}\left(\int_{\widetilde{\mathcal{P}}}\left|\frac{\widetilde{\chi}}{\varrho}\right|^{2} d x\right)^{\frac{1}{2}} .
\end{gathered}
$$


Combining estimates (4.9)-(4.12) and applying Young's inequality, we arrive at the final inequality

$$
\begin{aligned}
& \partial_{t} \int_{\widetilde{\mathcal{P}}}\left|\frac{\tilde{\chi}}{\varrho}\right|^{2} d x+\int_{\widetilde{\mathcal{P}}}\left|\nabla_{a}\left(\frac{\tilde{\chi}}{\varrho}\right)\right|^{2} d x \leq c \int_{\widetilde{\mathcal{P}}}|\nabla V|^{2} d x+ \\
& \quad+\left(\mathcal{A}_{2}^{2}+\mathcal{A}_{2} \int_{\widetilde{\mathcal{P}}}|\nabla V|^{2} d x\right)\left(\int_{\widetilde{\mathcal{P}}}\left|\frac{\widetilde{\chi}}{\varrho}\right|^{2} d x+1\right) .
\end{aligned}
$$

Estimate (4.13) implies

$$
\|\widetilde{\chi}\|_{L_{2, \infty}(\widetilde{Q})} \leq \Phi_{3}\left(\mathcal{A}_{2}\right)
$$

According to (4.6) and (4.7), one may conclude

$$
\int_{-2}^{2} \int_{1 / 4}^{3}\left|\nabla_{a} \widetilde{V}\right|^{2} d \varrho d x_{3} \leq c \int_{-2}^{2} \int_{1 / 4}^{3}\left(|\widetilde{\chi}|^{2}+\left|V^{a}\right|^{2}\right) d \varrho d x_{3} \leq \Phi_{3}\left(\mathcal{A}_{2}\right)
$$

and thus

$$
\int_{-2}^{2} \int_{1 / 4}^{3}|\widetilde{V}(x, t)|^{q} d \varrho d x_{3} \leq \Phi_{4}\left(q, \mathcal{A}_{2}\right)
$$

for all $t \in]-2^{2}, 0[$. Now, (4.2) immediately follows from the latter inequality. Lemma 4.2 is proved.

The second counterpart of the proof of Proposition 4.1 is the following statement.

Lemma 4.3 Under assumptions of Proposition 4.1, there exists a non decreasing function $\Phi_{5}: \mathbb{R}_{+} \rightarrow \mathbb{R}_{+}$such that

$$
\int_{\widetilde{Q}_{2}}\left|V_{\varphi}\right|^{6} d z \leq \Phi_{5}\left(\mathcal{A}_{2}\right)
$$

where $\left.\widetilde{Q}_{2}=\widetilde{\mathcal{P}}_{2} \times\right]-(3 / 2)^{2}, 0\left[\right.$ and $\widetilde{\mathcal{P}}_{2}=\mathcal{P}(3 / 8,5 / 2 ; 3 / 2)$. 
Proof We know that $V_{\varphi}$ satisfies the equation

$$
\begin{gathered}
\partial_{t} V_{\varphi}+V_{\varrho} V_{\varphi, \varrho}+V_{3} V_{\varphi, 3}+\frac{1}{\varrho} V_{\varrho} V_{\varphi} \\
-\left(V_{\varphi, \varrho \varrho}+V_{\varphi, 33}+\frac{1}{\varrho} V_{\varphi, \varrho}-\frac{1}{\varrho^{2}} V_{\varphi}\right)=0 .
\end{gathered}
$$

We fix a non-negative smooth and axially symmetric cut-off function $\psi$ vanishing in a neighborhood of the parabolic boundary of $\widetilde{Q}_{1}$ and being equal to 1 in $\widetilde{Q}_{2}$. Then, for $\widetilde{\alpha}=V_{\varphi} \psi \varrho$, we have the following identity

$$
\begin{gathered}
\partial_{t} \widetilde{\alpha}+V_{\varrho} \widetilde{\alpha}_{, \varrho}+V_{3} \widetilde{\alpha}_{, 3}-\left(\widetilde{\alpha}_{, \varrho \varrho}+\widetilde{\alpha}_{, 33}+\frac{1}{\varrho} \widetilde{\alpha}_{, \varrho}\right)+\frac{2}{\varrho} \widetilde{\alpha}_{, \varrho}= \\
=\alpha\left(\partial_{t} \psi+V_{\varrho} \psi_{, \varrho}+V_{3} \psi_{, 3}\right)-\left(2 \alpha_{, \varrho} \psi_{, \varrho}+2 \alpha_{, 3} \psi_{, 3}+\alpha \psi_{, \varrho \varrho}+\alpha \psi_{, 33}\right)+\frac{1}{\varrho} \alpha \psi_{, \varrho},
\end{gathered}
$$

where $\alpha=V_{\varphi} \varrho$.

Then, we multiply the latter identity by $\widetilde{\alpha}|\widetilde{\alpha}|^{2}$ and integrate the product by parts over $\widetilde{\mathcal{P}}_{1}$

$$
\frac{1}{4} \partial_{t} \int_{\widetilde{\mathcal{P}}_{1}}|\widetilde{\alpha}|^{4} d x+\frac{3}{4} \int_{\widetilde{\mathcal{P}}_{1}}\left|\nabla_{a}\left(|\widetilde{\alpha}|^{2}\right)\right|^{2} d x=J_{1}+J_{2}
$$

where

$$
J_{1}=\int_{\widetilde{\mathcal{P}}_{1}} \alpha \widetilde{\alpha}|\widetilde{\alpha}|^{2}\left(V_{\varrho} \psi, \varrho+V_{3} \psi_{, 3}\right) d x
$$

and

$$
J_{2}=\int_{\widetilde{\mathcal{P}}_{1}} \widetilde{\alpha}|\widetilde{\alpha}|^{2}\left(\alpha \partial_{t} \psi-2 \alpha_{, \varrho} \psi_{, \varrho}-2 \alpha_{, 3} \psi_{, 3}-\alpha \psi_{, \varrho \varrho}-\alpha \psi_{, 33}+\frac{1}{\varrho} \alpha \psi_{, \varrho}\right) d x .
$$

We let $\beta=|\widetilde{\alpha}|^{2}$, then $|\beta|^{\frac{10}{3}}=|\widetilde{\alpha}|^{\frac{20}{3}}$ and

$$
\int_{\widetilde{\mathcal{P}}_{1}}|\beta|^{\frac{10}{3}} d x \leq c\left(\int_{\widetilde{\mathcal{P}}_{1}}|\beta|^{2} d x\right)^{\frac{2}{3}} \int_{\widetilde{\mathcal{P}}_{1}}|\nabla \beta|^{2} d x \text {. }
$$


We start with $J_{1}$, setting $\mathcal{A}_{3}=\left\|V^{a}\right\|_{L_{4, \infty}\left(\widetilde{Q}_{1}\right)}$. By Hölder's inequality and by multiplicative inequality (4.17),

$$
\begin{gathered}
J_{1} \leq c\left(\int_{\widetilde{\mathcal{P}}_{1}}|\widetilde{\alpha}|^{\frac{20}{3}} d x\right)^{\frac{9}{20}}\left(\int_{\widetilde{\mathcal{P}}_{1}}|\alpha|^{\frac{20}{11}}\left|V^{a}\right|^{\frac{20}{11}} d x\right)^{\frac{11}{20}} \leq \\
\leq c\left(\int_{\widetilde{\mathcal{P}}_{1}}|\beta|^{\frac{10}{3}} d x\right)^{\frac{9}{20}}\left(\int|\alpha|^{\frac{10}{3}} d x\right)^{\frac{3}{10}}\left(\int_{\widetilde{\mathcal{P}}_{1}}\left|V^{a}\right|^{4} d x\right)^{\frac{1}{4}} \leq \\
\leq c\left(\int_{\widetilde{\mathcal{P}}_{1}}|\beta|^{\frac{10}{3}} d x\right)^{\frac{9}{20}}\left(\int_{\widetilde{\mathcal{P}}_{1}}|V|^{2} d x\right)^{\frac{1}{5}}\left(\int_{\widetilde{\mathcal{P}}_{1}}|V|^{2} d x+\int_{\widetilde{\mathcal{P}}_{1}}|\nabla V|^{2} d x\right)^{\frac{3}{10}} \mathcal{A}_{3} \leq \\
\leq c\left(\int_{\widetilde{\mathcal{P}}_{1}}|\beta|^{\frac{10}{3}} d x\right)^{\frac{9}{20}} \mathcal{A}_{2}^{\frac{1}{5}}\left(\int_{\widetilde{\mathcal{P}}_{1}}|\nabla V|^{2} d x+\mathcal{A}_{2}\right)^{\frac{3}{10}} \mathcal{A}_{3} \leq \\
\leq c\left(\int_{\widetilde{\mathcal{P}}_{1}}|\beta|^{2} d x\right)^{\frac{3}{10}}\left(\int_{\widetilde{\mathcal{P}}_{1}}|\nabla \beta|^{2} d x\right)^{\frac{9}{20}} \mathcal{A}_{2}^{\frac{1}{5}}\left(\int_{\widetilde{\mathcal{P}}_{1}}|\nabla V|^{2} d x+\mathcal{A}_{2}\right)^{\frac{3}{10}} \mathcal{A}_{3} .
\end{gathered}
$$

Term $J_{2}$ is estimated in the same way:

$$
\begin{aligned}
& J_{2} \leq c\left(\int_{\widetilde{\mathcal{P}}_{1}}|\widetilde{\alpha}|^{\frac{20}{3}} d x\right)^{\frac{9}{20}}\left(\int_{\widetilde{\mathcal{P}}_{1}}\left(|\alpha|+\left|\alpha_{, \varrho}\right|+\left|\alpha_{, 3}\right|\right)^{\frac{20}{11}} d x\right)^{\frac{11}{20}} \leq \\
& \leq c\left(\int_{\widetilde{\mathcal{P}}_{1}}|\beta|^{2} d x\right)^{\frac{3}{10}}\left(\int_{\widetilde{\mathcal{P}}_{1}}|\nabla \beta|^{2} d x\right)^{\frac{9}{20}}\left(\int_{\widetilde{\mathcal{P}}_{1}}|\nabla V|^{2} d x+\mathcal{A}_{2}\right)^{\frac{1}{2}} .
\end{aligned}
$$

Now, making use of Young's inequality, we derive from (4.17) and from two latter estimates the main inequality

$$
\begin{gathered}
\partial_{t} \int_{\widetilde{\mathcal{P}}_{1}}|\widetilde{\alpha}|^{4} d x+\int_{\widetilde{\mathcal{P}}_{1}}\left|\nabla_{a}\left(|\widetilde{\alpha}|^{2}\right)\right|^{2} d x \leq \\
\leq c\left(\int_{\widetilde{\mathcal{P}}_{1}}|\beta|^{2} d x\right)^{\frac{6}{11}} \mathcal{A}_{2}^{\frac{4}{11}}\left(\int_{\widetilde{\mathcal{P}}_{1}}|\nabla V|^{2} d x+\mathcal{A}_{2}\right)^{\frac{6}{11}} \mathcal{A}_{3}^{\frac{20}{11}}
\end{gathered}
$$




$$
\begin{gathered}
+c\left(\int_{\widetilde{\mathcal{P}}_{1}}|\beta|^{2} d x\right)^{\frac{6}{11}}\left(\int_{\widetilde{\mathcal{P}}_{1}}|\nabla V|^{2} d x+\mathcal{A}_{2}\right)^{\frac{10}{11}} \leq \\
\leq c \int_{\widetilde{\mathcal{P}}_{1}}|\beta|^{2} d x\left(\int_{\widetilde{\mathcal{P}}_{1}}|\nabla V|^{2} d x+\mathcal{A}_{2}\right)+c\left(\mathcal{A}_{2} \mathcal{A}_{3}^{5}\right)^{\frac{4}{11}}+c\left(\int_{\widetilde{\mathcal{P}}_{1}}|\nabla V|^{2} d x+\mathcal{A}_{2}\right)^{\frac{4}{11}} .
\end{gathered}
$$

It, together with the statement of Lemma 4.2 at $q=4$, implies

$$
\sup _{-(7 / 4)^{2} \leq t \leq 0} \int_{\widetilde{\mathcal{P}}_{1}}|\beta(x, t)|^{2} d x+\int_{\widetilde{Q}_{1}}|\nabla \beta|^{2} d z \leq \Phi_{5}\left(\mathcal{A}_{2}\right) .
$$

So, (4.14) follows from (4.17) and (4.18). Lemma 4.3 is proved.

From Lemmata 4.2 and 4.3 , we find

Corollary 4.4 Under assumptions of Proposition 4.1, there exists a nondecreasing function $\Phi_{6}: \mathbb{R}_{+} \rightarrow \mathbb{R}_{+}$such that

$$
\int_{\widetilde{Q}_{2}}|V|^{6} d z \leq \Phi_{6}\left(\mathcal{A}_{2}\right) \text {. }
$$

Proof of Proposition 4.1 Applying Corollary 4.4 and Lemma 2.3, we end up with the proof of Proposition 4.1. Proposition 4.1] is proved.

\section{Proof of Theorem 1.2}

Given $R>1$, let us consider the following space-time cylinder

$$
\left.\widetilde{Q}_{R}^{b}=\widetilde{\mathcal{P}}_{R}^{b} \times\right]-(2 R)^{2}, 0[
$$

where $b \in \mathbb{R}$ and

$$
\widetilde{\mathcal{P}}_{R}^{b}=\widetilde{\mathcal{P}}_{R}+b e_{3}, \quad \widetilde{\mathcal{P}}_{R}=\mathcal{P}(R / 4,3 R ; 2 R) .
$$

Now, we scale our blow up functions $u$ and $q$ in the following way

$$
u^{R}(x, t)=R u\left(R x+b e_{3}, R^{2} t\right), \quad q^{R}(x, t)=R^{2} q\left(R x+b e_{3}, R^{2} t\right)
$$

for $z=(x, t) \in \widetilde{Q}$. 
Functions $u^{R}$ and $q^{R}$ are axially symmetric and, as it was explained before, sufficiently smooth to apply Proposition 4.1. According to that, we have

$$
\sup _{z \in \widetilde{Q}_{0}}\left\{\left|u^{R}(z)\right|+\left|\nabla u^{R}(z)\right|\right\} \leq \Phi\left(\mathcal{A}_{2}\right)
$$

where $\widetilde{Q}_{0}=\mathcal{P}(1,2 ; 1)$ and

$$
\mathcal{A}_{2}=\sup _{-2^{2} \leq t \leq 0} \int_{\widetilde{\mathcal{P}}}\left|u^{R}(x, t)\right|^{2} d x+\int_{\widetilde{Q}}\left(\left|\nabla u^{R}\right|^{2}+\left|u^{R}\right|^{3}+\left|q^{R}\right|^{\frac{3}{2}}\right) d z .
$$

Then, we make the inverse change of variables. As a result, we find

$$
\sup _{(y, s) \in Q_{R}^{b}}\left\{R|u(y, s)|+R^{2}|\nabla u(y, s)|\right\} \leq \Phi\left(\widetilde{\mathcal{A}}_{2 R}\right),
$$

where $\left.Q_{R}^{b}=\widetilde{\mathcal{P}}_{0 R}^{b} \times\right]-R^{2}, 0\left[, \widetilde{\mathcal{P}}_{0 R}^{b}=b e_{3}+\mathcal{P}_{0 R}, \mathcal{P}_{0 R}=\mathcal{P}(R, 2 R ; R)\right.$, and

$$
\begin{gathered}
\widetilde{\mathcal{A}}_{2 R}=\sup _{-(2 R)^{2} \leq s \leq 0} \frac{1}{R} \int_{-2 R+b}^{2 R+b} d y_{3} \int_{R / 4<\left|y^{\prime}\right|<3 R}|u(y, s)|^{2} d y^{\prime}+ \\
+\frac{1}{R} \int_{-(2 R)^{2}}^{0} d s \int_{-2 R+b}^{2 R+b} d y_{3} \int_{R / 4<\left|y^{\prime}\right|<3 R}|\nabla u(y, s)|^{2} d y^{\prime}+ \\
+\frac{1}{R^{2}} \int_{-(2 R)^{2}}^{0} d s \int_{-2 R+b}^{2 R+b} d y_{3} \int_{R / 4<\left|y^{\prime}\right|<3 R}\left(|u(y, s)|^{3}+|q(y, s)|^{\frac{3}{2}}\right) d y^{\prime} \leq \\
\leq c\left(A\left(e^{b}, 3 R ; u\right)+E\left(e^{b}, 3 R ; u\right)+C\left(e^{b}, 3 R ; u\right)+D\left(e^{b}, 3 R ; q\right)\right) \leq c \mathcal{A},
\end{gathered}
$$

$e^{b}=\left(y^{b}, 0\right)$ and $y^{b}=(0, b)$. So, assuming that $\left|y^{\prime}\right|>20$, we can derive from the latter estimates

$$
\left|y^{\prime}\right|\left|u\left(y^{\prime}, b, s\right)\right|+\left|y^{\prime}\right|^{2}\left|\nabla u\left(y^{\prime}, b, s\right)\right| \leq \Phi(c \mathcal{A})
$$

for any $b \in \mathbb{R}$, for any $\left|y^{\prime}\right|>20$, and for any $s \in[-20,0]$. It follows directly from (5.1) that:

$$
|u(y, s)|+|\nabla u(y, s)| \leq c \Phi(c \mathcal{A})=c(\mathcal{A})
$$


for any $\left|y^{\prime}\right|>20$ and for any $s \in[-20,0]$.

Now, we consider the vorticity $\omega(u)=\nabla \wedge u$. It satisfies the vorticity equation

$$
\partial_{t} \omega-\Delta \omega=\omega \cdot \nabla u-u \cdot \nabla \omega
$$

which, together with 5.2, implies

$$
\left|\partial_{t} \omega-\Delta \omega\right| \leq c(\mathcal{A})(|\omega|+|\nabla \omega|)
$$

for any $\left|y^{\prime}\right|>20$ and for any $s \in[-20,0]$. Moreover, by (3.11),

$$
\omega(\cdot, 0)=0 \quad \text { in } \quad \mathbb{R}^{3} .
$$

By the backward uniqueness results for the heat operator with variable lower order terms in a half-space, see [3], 4], and [15], and, by (5.3) and (5.4), we state

$$
\omega(y, s)=0
$$

for any $\left|y^{\prime}\right|>20$ and for any $s \in[-20,0]$.

Since our solution is sufficiently smooth in $\mathbb{R}^{3} \backslash\left\{y^{\prime} \neq 0\right\} \times[-10,0]$, one can make use of the unique continuation through spatial boundaries and conclude that

$$
\nabla \wedge u \equiv 0 \quad \text { in } \quad \mathbb{R}^{3} \backslash\left\{y^{\prime} \neq 0\right\} \times[-8,0] .
$$

On the other hand, from (3.8), it follows that

$$
\mathcal{A}_{0} \geq \operatorname{ess} \sup _{-20 \leq s \leq 0} \int_{\left|y^{\prime}\right| \leq 40} \frac{|u(y, s)|^{2}}{\left|y^{\prime}\right|} d y .
$$

So, we observe that, for any $s \in S$,

$$
\int_{-\infty}^{+\infty} d y_{3} \int_{\left|y^{\prime}\right| \leq 40} \frac{|u(y, s)|^{2}}{\left|y^{\prime}\right|} d y^{\prime} \leq \mathcal{A}_{0}<+\infty
$$

where $S \subset[-20,0]$ and $|S|=20$.

Now, we wish to show

$$
\nabla \wedge u(\cdot, s) \equiv 0 \quad \text { in } \quad \mathbb{R}^{3}
$$


for any $s \in S$. To this end, we proceed as follows. Let $\varphi \in C_{0}^{\infty}\left(B^{\prime}\right)$ be a non-negative cut-off function being equal to 1 in $B^{\prime}(1 / 2)$. Here, $B^{\prime}$ and $B^{\prime}(1 / 2)$ are two-dimensional balls centered at the origin with radii 1 and $1 / 2$, respectively. Next, let $\psi$ be an arbitrary smooth, compactly supported in $\mathbb{R}^{3}$, vector-valued function. Then, by (5.6), for any $s \in[-8,0]$,

$$
\begin{gathered}
\int_{\mathbb{R}^{3}} u(y, s) \cdot \nabla \wedge\left(\psi(y)\left(1-\varphi\left(y^{\prime} / R\right)\right)\right) d y=0= \\
=\int_{\mathbb{R}^{3}} u(y, s) \cdot \nabla \wedge \psi(y) d y-\int_{\mathbb{R}^{3}} u(y, s) \cdot \nabla \wedge\left(\psi(y) \varphi\left(y^{\prime} / R\right)\right) d y=J_{1}(s)+J_{2}(s) .
\end{gathered}
$$

For $J_{2}$, we have the estimate

$$
\begin{gathered}
\left|J_{2}(s)\right| \leq c\left(1+\frac{1}{R}\right) \int_{\operatorname{spt} \psi \cap\left\{\left|y^{\prime}\right|<R\right\}}|u(y, s)| d y= \\
=c\left(1+\frac{1}{R}\right) \int_{\operatorname{spt} \psi \cap\left\{\left|y^{\prime}\right|<R\right\}} \frac{|u(y, s)|}{\left|y^{\prime}\right|^{\frac{1}{2}}}\left|y^{\prime}\right|^{\frac{1}{2}} d y \leq \\
\leq c\left(1+\frac{1}{R}\right)\left(\int_{\operatorname{spt} \psi \cap\left\{\left|y^{\prime}\right|<R\right\}} \frac{|u(y, s)|^{2}}{\left|y^{\prime}\right|} d y\right)^{\frac{1}{2}}\left(\int_{\operatorname{spt} \psi \cap\left\{\left|y^{\prime}\right|<R\right\}}\left|y^{\prime}\right| d y\right)^{\frac{1}{2}} \leq \\
\leq c(\psi)\left(1+\frac{1}{R}\right) R^{\frac{3}{2}}\left(\int_{-\infty}^{+\infty} d y_{3} \int_{\left|y^{\prime}\right|<40} \frac{|u(y, s)|^{2}}{\left|y^{\prime}\right|} d y^{\prime}\right)^{\frac{1}{2}} .
\end{gathered}
$$

By (5.7), the right hand side of the latter inequality goes to zero as $R \rightarrow 0$ for any $s \in S$. Hence, $J_{1}(s)=0$ for any $s \in S \cap[-8,0]$, which is but a weak form of (5.8). By the fact that $u$ is divergence free, we then show

$$
\Delta u(\cdot, s)=0 \quad \text { in } \quad \mathbb{R}^{3}
$$

for any $s \in S \cap[-8,0]$.

Now, let $B\left(y_{0}, R\right)$ be a ball of radius $R$ with the center at the point $y_{0}$. For any $y_{0} \in\left\{\left|y^{\prime}\right| \leq 30, y_{3} \in \mathbb{R}\right\}$,

$$
B\left(y_{0}, 1\right) \subset\left\{\left|y^{\prime}\right| \leq 40, y_{3} \in \mathbb{R}\right\}
$$


and, since $u$ is harmonic,

$$
\begin{gathered}
\left|u\left(y_{0}, s\right)\right| \leq c\left(\int_{B\left(y_{0}, 1\right)}|u(y, s)|^{2} d y\right)^{\frac{1}{2}} \leq c\left(\int_{\left|y^{\prime}\right| \leq 40}|u(y, s)|^{2} d y\right)^{\frac{1}{2}} \leq \\
\leq c \sqrt{40 \mathcal{A}_{0}}
\end{gathered}
$$

for any $s \in S \cap[-8,0]$. So, according to (5.2), the function $u(\cdot, s)$ is bounded in $\mathbb{R}^{3}$ for any $s \in S \cap[-8,0]$. But, by (5.1), in fact, $u(\cdot, s)=0$ in $\mathbb{R}^{3}$ for any $s \in S \cap[-8,0]$. This contradicts with (3.9). Theorem 1.2 is proved.

\section{References}

[1] Caffarelli, L., Kohn, R.-V., Nirenberg, L., Partial regularity of suitable weak solutions of the Navier-Stokes equations, Comm. Pure Appl. Math., Vol. XXXV (1982), pp. 771-831.

[2] Chae D., Lee, J., On the regularity of the axisymmetric solutions of the Navier-Stokes equations, Math. Z., 239(2002), 645-671.

[3] Escauriaza,L., Seregin, G., Šverák, V., Backward uniqueness for the heat operator in half space, Algebra and Analyis, 15(2003), no. 1, 201214.

[4] Escauriaza,L., Seregin, G., Šverák, V.,. $L_{3, \infty}$-Solutions to the NavierStokes equations and backward uniqueness, Uspekhi Matematicheskih Nauk, v. 58, 2(350), pp. 3-44. English translation in Russian Mathematical Surveys, 58(2003)2, pp. 211-250.

[5] Giga, Y., Solutions for semilinear parabolic equations in $L^{p}$ and regularity of weak solutions of the Navier-Stokes equations, J. of Diff. Equations, 62(1986), pp. 186-212.

[6] Ladyzhenskaya, O. A., On uniqueness and smoothness of generalized solutions to the Navier-Stokes equations, Zapiski Nauchn. Seminar. POMI, 5(1967), pp. 169-185.

[7] Ladyzhenskaya, O. A., On unique solvability of the three-dimensional Cauchy problem for the Navier-Stokes equations under the axial symmetry, Zap. Nauchn. Sem. LOMI 7(1968), 155-177. 
[8] Ladyzhenskaya, O. A., Seregin, G. A., On partial regularity of suitable weak solutions to the three-dimensional Navier-Stokes equations, J. math. fluid mech., 1(1999), pp. 356-387.

[9] Leonardi, S., Malek, Necas, J., \& Pokorny, M., On axially simmetric flows in $\mathbb{R}^{3}$, ZAA, 18(1999), 639-649.

[10] Lin, F.-H., A new proof of the Caffarelly-Kohn-Nirenberg theorem, Comm. Pure Appl. Math., 51(1998), no.3, pp. 241-257.

[11] Neustupa, J., Pokorny, M., Axisymmetric flow of Navier-Stokes fluid in the whole space with non-zero angular velocity compnents, Math. Bohemica, 126(2001), 469-481.

[12] Pokorny, M., A regularity criterion for the angular velocity component in the case of axisymmetric Navier-Stokes equations, 2001.

[13] Prodi, G., Un teorema di unicità per el equazioni di Navier-Stokes, Ann. Mat. Pura Appl., 48(1959), pp. 173-182.

[14] Seregin, G. A. On the number of singular points of weak solutions to the Navier-Stokes equations, Comm. Pure Appl. Math., 54(2001), issue 8, pp. 1019-1028.

[15] Seregin, G.A., On smoothness of $L_{3, \infty}$-solutions to the Navier-Stokes equations up to boundary, Mathematische Annalen, 332(2005), pp. 219238.

[16] Seregin, G., New version of the Ladyzhenskaya-Prodi-Serrin condition, Algebra i Analiz, 18(2006), 124-143.

[17] Seregin, G., Local regularity theory of the Navier-Stokes equations, to appear in Handbook of Mathematical Fluid Mechanics, vol. 4.

[18] Seregin, G., Estimates of suitable weak solutions to the Navier-Stokes equations in critical Morrey spaces, Zapiski Nauch. Seminar POMI, 2006.

[19] Serrin, J., On the interior regularity of weak solutions of the NavierStokes equations, Arch. Ration. Mech. Anal., 9(1962), pp. 187-195. 
[20] Struwe, M., On partial regularity results for the Navier-Stokes equations, Comm. Pure Appl. Math., 41 (1988), no. 4, 437-458.

[21] Ukhovskij, M. R., Yudovich, V. L., Axially symmetric motions of ideal and viscous fluids filling all space, Prikl. Mat. Mech. 32 (1968), 59-69.

[22] Wiegner, M., Zajaczkowski, W. M., On stability of axially symmetric solutions to Navier-Stokes equations in a cylindrical domain and with boundary slip conditions, Banach Center Publ., 70(2005), 251-278.

[23] Zajaczkowski, W. M., Global special regular solutions to the NavierStokes equations in axially symmetric domains under boundary slip conditions, Diss. Math., 400(2005).

[24] Zajaczkowski, W. M., Global special regular to the Navier-Stokes equations in a cylindrical domain under boundary slip conditions, Gakuto Series in Mathematics, vol. 21, 2004.

G. Seregin

Steklov Institute of Mathematics at St.Petersburg, St.Petersburg, Russia

W.Zajaczkowski

Institute of Mathematics, Polish Academy of Sciences, Sniadeckich 8, 00-956 Warsaw, Poland 